\title{
25 Research Square

\section{Population's unmet needs in relation to primary care in Romania- a pilot survey in County Brasov.}

Andrea Elena Neculau ( $\square$ andrea.neculau@gmail.com )

Universitatea Transilvania din Brasov Facultatea de Medicina https://orcid.org/0000-0002-4031-5694

\section{Liliana Rogozea}

Universitatea Transilvania din Brasov Facultatea de Medicina

\section{Daniela Popa}

Universitatea Transilvania din Brasov

Ioana Atudorei

Universitatea Transilvania din Brasov

\section{Florin Leasu}

Universitatea Transilvania din Brasov Facultatea de Medicina

Marius Alexandru Moga

Universitatea Transilvania din Brasov Facultatea de Medicina

Anca Lacatus

Universitatea Transilvania din Brasov Facultatea de Medicina

\section{Research article}

Keywords: unmet health needs, primary care, Romania

Posted Date: September 26th, 2019

DOI: https://doi.org/10.21203/rs.2.14590/v1

License: (c) (i) This work is licensed under a Creative Commons Attribution 4.0 International License.

Read Full License 


\section{Abstract}

Background Patients' expectations and needs for healthcare services are changing. These changes are correlated with changes in disease profiles, a higher prevalence of chronic diseases, the introduction of new and innovative treatments and health technologies, and the emergence of new social and economic contexts. National health reports on Romania show that decisions in healthcare planning are not correlated with the health needs of the population. At the same time, this report shows a high degree of unmet healthcare needs of the Romanian population (related to cost, distance and waiting times), especially for low-income populations. The objective of the study was to identify the unmet needs of the population in relation to primary care medical services in the context of actual health regulations through a pilot study in a representative county in Romania. Methods The study is survey-based, and part of a health needs assessment programme commissioned by the District Health Authority to the university. A questionnaire with 21 items was designed to gather information about the structures, processes and outcomes of primary care from the perspective of the population. A total of 877 questionnaires were returned and validated. The data were analysed with SPSS version 25. Results Access to primary care was considered to be good by most of the population. Most of the settlements have a family doctor, and $80.5 \%$ can schedule an appointment on the same or the following day. Most basic medical services are provided, except for out-of-hours primary care services and cervical cancer screening. The family doctors are considered to be a reliable health resource. Conclusions Despite limitations in the practice of family medicine in Romania and therefore a narrow spectrum of services offered by primary care in general, the level of contentment of the population with this healthcare resource is still high. Barriers to access are related to the lack of some essential services, especially preventive and out-of-hours services. Unmet needs are presumably not recognised by patients due to a lack of medical culture. Further research is needed to clarify this conclusion. Key words: unmet health needs, primary care, Romania

\section{Background}

Patients expectations and needs from healthcare services are changing. It is correlated with the change of the profile of diseases, with a higher prevalence of chronic ones, the introduction of new and innovative treatments and health technologies, but also with the emergence of new social and economic contexts. Healthcare systems need to adapt to these trends and aim to cover the health needs of the population. Where does Romania stand in this equation especially in relation to primary care is a question worse to respond.

In 2017, The European Observatory on Health System and Polices has published a National report on Romania, showing that, while Romanians claim to have a good health, mortality figures are still very high, being among the first in Europe for most preventable diseases ${ }^{1}$. 
The underfinancing of the Healthcare system is one of the main causes identified by the authors of the report, but also the inefficient use of resources. Healthcare planning is merely top down and is not correlated with the health needs of the population, Romania is reporting a high degree of unmet Healthcare needs of the population related to cost, distance and waiting times, especially for the low-income group ${ }^{2}$.

Primary care is an important segment of the Healthcare system. In terms of health policies, it is entirely assimilated to Family Medicine. The context of practice in family medicine in Romania has certain particularities. It contains both elements favouring access and equity but also important barriers to it. One of the enablers to access is the fact that, in Romania, nearly all patients are registered with a family doctor (FD), the latter having a gatekeeping role. At this level, free consultations are offered for all insured people (more than $85 \%)^{2}$. The uninsured have also access to a minimal package of services. The free choice of the FD is also guaranteed by the National Health Insurance Contract.

Certain barriers to the practice of FDs are present. Due to cost control policies, protocols of prescription have been used to limit the initiation of certain medication. Medication for type 2 diabetes or asthma inhalers cannot be prescribed by FDs, a referral to the specialist being needed. Other limitations are present in the reimbursement of procedures, discouraging FDs form performing them. In this context, we are registering a narrowing of the scope of care of family medicine, this one being transformed into a referral point and not an intervention one, in the system.

The question arises if there is still a recognized role of primary care in the community and what expectations our patients still have in relationship to it. The analyses of unmet health care needs allow to explore to what degree system barriers have altered the use of medical services in primary care, the trust in FD's and ultimately in the role itself of the FD in the community.

\section{Methods}


The research consisted in a survey, performed in the context of a Health Needs Assessment Project commissioned by the County Council of Brasov to Transylvania University. The survey was applied to a representative sample of the population (1200) of County Brasov Romania that accounts for 634.236 inhabitants.

A theoretical sampling with mapping of all representative communities was considered. The statistical error of the sample is $3,32 \%$ for $\mathrm{p}=95 \%$.

In the project, we have designed a 54 items questionnaire, meant to analyse the felt and expressed needs of the population and the need to access different levels of healthcare in the past 12 months. 21 of these questions were dedicated to exploring for possible unmet health needs at the level of primary care. The questionnaire had preformulated answers that where prefiguring recognised normative aspect of care. It was self-administered. The location of delivery were FDs practices, local Councils and other places in the community. 877 questionnaires were returned and validated.

The Alpha Cronbach coefficient is 0,828 a value that has allowed us to pursue research.

Questions where grouped to explore the three dimensions of care: structure, process and outcomes of care ${ }^{3}$.

Structure attributes have targeted the following: waiting time till appointment, opening hours, access by telephone, availability of a FDs in the community, presence of a second family doctor in the community, availability of Out of Hours services led by FD's.

Process attributes: Continuity of care, Information on medication, information on prevention, time spent at consultation, span of diseases that can be addressed, availability of preventive services (Pap smear), medical procedures available (blood draw/ electrocardiogram), knowledge of FD about patient's history, facilitation of access to secondary or tertiary care. Outcomes of care attributes: health problem not resolved after FD encounter, better informed on health problem after visit at the FD. 
We have also explored some patterns of relating with family medicine like the number of visits at the family doctor per year, the constancy in being registered with FD.

Data was analysed with SPSS version 25.

\section{Results}

The demographic characteristics of the sample are summarized in table1. It is to be noticed that the population over 65 is higher $(18,2 \%)$ than the average of the County $(15,8 \%)$ as published by the County Statistical Department. Gender distribution is favouring women, $67,2 \%$ female and $32,8 \%$ male. The level of education is as following: $11,6 \%$ have a basic education level (8 classes and under), 61,1\% have graduated a high school or a professional school and 27,2\% have a university background. This distribution is covering the broad spectrum of education in the community.

In terms of visits to the FD, 15,6\% of patients have visited their FD once in the past 12 months and 19,8\% have visited the FD for 12 times 12 months and only 3,9\% more than 12 times.

Table 1. Demographic data of the sample 


\begin{tabular}{|c|c|c|c|c|c|}
\hline Data & Category & & & & \\
\hline \multirow[t]{3}{*}{ Age distribution (\%) } & $18-30 y$ & $31-50 y$ & & $51-65 y$ & Over \\
\hline & & & & & $65 y$ \\
\hline & 19,7 & 39 & & 22,4 & 18,9 \\
\hline \multirow[t]{2}{*}{ Gender distribution (\%) } & Female & Male & & & \\
\hline & 67,2 & 32,8 & & & \\
\hline \multirow[t]{3}{*}{ Level of education (\%) } & Primary school & Professional & High School & University and & \\
\hline & & & & more & \\
\hline & 11,6 & 13,7 & 47,4 & 27,2 & \\
\hline \multirow[t]{2}{*}{ Residence (\%) } & Urban & Rural & & & \\
\hline & 48 & 52 & & & \\
\hline \multirow[t]{3}{*}{ Years with same FD } & Less than 10 & More than 10 & & & \\
\hline & years & years & & & \\
\hline & $46,4 \%$ & $53,6 \%$ & & & \\
\hline Number of visits with & One & Less than 12 & More than & Average number & \\
\hline \multirow[t]{3}{*}{ FD in the past 12 Months } & & & 12 & & \\
\hline & & & & & \\
\hline & 15,6 & 19,8 & 3,9 & 11,25 & \\
\hline
\end{tabular}

The study was a typical approach to Health needs assessment (HNA). HNA is recognised as a method to identify gaps in care. Health needs are various: felt needs, expressed needs, 
normative needs ${ }^{4}$. All of these can be met or unmet at a certain moment, in correlation with the three dimensions: need, supply and demand. The patient is central to this equation and not always consulted.

Our study is offering the responses of a significant sample of the population of the County showing the positive and the negative parts of primary care as it is perceived by the population.

\section{Access to primary care}

In Romania, in the framework of the National Health Insurance System (NHIS), nearly all patients are registered with a FD. The main limitation of access to a FD is the lack of family doctors in certain regions. In Brasov County only 2,6\% of patients declared that they do not have a FD in the area where they live. In other regions of Romania this problem is bigger. In total, after an estimation made by professional organizations in 2014, there is a lack of over 500 GPs in Romania (unofficial data). The phenomenon of the deficit of professional resources in family Medicine is known worldwide and policies to stimulate retention of this workforce should be developed especially by local communities.

Asked if reaching their FD was a problem (in the past 6 months) 88,6\% of the population answered not having had this problem and only 11,6\% answered that they encountered problems. In comparison with other types of specialists, accessing FD's seems not to be a problem. Responders signalled difficulties in reaching an eye specialist (37,3\% couldn't reach in the past 6 month) or a cardiologist (43,1\% couldn't reach in the past 6 month).

Furthermore, the results of our study showed that only $26,6 \%$ of people could get a same day appointment with their GP. This is lower than the report of the Regional WHO office from 2009 that showed that $92,8 \%$ of people could get same day appointment with the FD ${ }^{5}$.

Nevertheless, our study is showing that next day appointment is possible in $53,9 \%$ of cases. 
Being asked if there was an alternative doctor to see if their FD is missing, in 27,6\% of cases, people affirmed that there was no other doctor to replace their FD in the community.

In only $58,6 \%$ of cases there is access to an Out- of- Hour $(\mathrm{OOH})$ centre in the neighbourhood.

Opening hours of FDs offices allow most people (91,2\%) to access the practice. Access to FD's office by telephone is available in $90,5 \%$ of practices.

Although in a study published in 2015 data showed only occasional access by telephone and or to an appointment system in the FDs office, this situation has changed in the past years, since the introduction of a compulsory appointment system by the National Health Insurance House $(\mathrm{NHIH})^{6}$.

Table 2. Health needs in relation to access to the FD's office 

or her?

In the place you live the opening hours of the FD allow you to access it whenever you need it?

In the place you live is there an Out-of- Hours centre?

\section{Longitudinal continuity of care}

Our study showed that patients prefer long-term relationship with their FD, even though there is a possibility for the free choice of the doctor. Most patients are registered with their FD for more than 10 years $(53,6 \%)$. 


\section{Seeking for medical care at the FD}

The average number of visits at the FD in the past 12 months in our study was 11.25. Most patients visited their FD for 12 times (19,8\%). People aged more than 65 are the most frequent accessors at the FD (Figure 1). A European data base ${ }^{7}$ (Eurostat, Healthcare activities statistics- consultations) is showing an average of 5.7 contacts with the GP and a National report from 2009 is showing 7.7 visits per years ${ }^{5}$. The increase in number of visits is due to the Contract Framework of the National Health Insurance House (NHIH) that is specifying the gatekeeping role of the FD.

Figure 1. Seeking for medical care at the FD according to age

Limitations of access due to system organization

- Limited number of consultations per day

Although the number of payed consultations in the contract is limited to 20 (24) a day, FD's are covering extra patients every day reducing the length of the consultations that is normally set to 15 minutes per patient.

- Limited opening hours

In Romania FD's offices are open only 5-6 hours a day, many practices working in alternative shifts (morning and afternoon). The results of the study are showing that people do not find this as a barrier of access, although same day visit is available only in $26,6 \%$ of cases. In our opinion it is probably linked with the lack of another perspective. If alternative model practices, with more time availability, could be an option, perhaps patient's options would have been more differentiated.

- Access to continuity of care - Out-of - Hours $(\mathrm{OOH})$ and or other health resources 
Access to Out-of-Hours services is a problem due to the lack of coverage in the hole territory of the County. It is a result of the fact that $\mathrm{OOH}$ is a service organized by FD's at their one decision, without a rigorous planning of the service by the District Health Authorities. In $27,6 \%$ of cases the FD is the only health resource in the community. Community and school nurses are unavailable in most of the rural communities.

\section{Person centred care and trust ( Table 3)}

In terms of the process of care, responders have shown that they consider FDs a reliable health resource. The FD who knows their history and medication, can inform and educate them in relation with their disease. This result is showing that even though there is a reduced variety of services that FDs can provide (in the situations of the restrictive contractual framework) patients are still counting on their FD's. The potential of this relationship is important, and FD's are to be encouraged to practice at their full potential, broadening the spectrum of services that they deliver to patients, especially those that are important to public health.

Table 3. Health needs in relation to the processes of care in FD's office 
Does your FD know the history of your diseases?

Does your FD know the history of medication you take?
$93,8 \quad 6,2$

$93,5 \quad 6,5$

Did you receive clear information from your FD regarding your illness? $\quad 88,8 \quad 11,2$

Did you receive clear information regarding the way to prevent illnesses? $\quad 87,7 \quad 11,2$

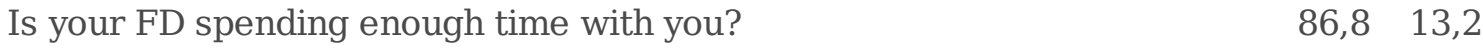

At the FD you can address any medical problem? $\quad 87,6 \quad 12,4$

In FD's office can you get facilitation to the appointments for $\quad 82,1 \quad 17,9$

Secondary or tertiary care

\section{Health improvement and education ( Table 4)}

Results of our study are showing that most of the responders are satisfied with results of care.

Health education and medical advice is recognized to be valuable in $88,8 \%$ of cases concerning diseases and $87,7 \%$ for preventative measures.

$52,3 \%$ of patients suffered from a chronic disease and $91,9 \%$ of them affirmed that they understood form medical advice of their FD how to take this medication.

Table 4. Health needs in relation to outcomes of care in FD's office 


\section{Availability of procedures ( Table 5)}

We looked at some of the procedures relevant to public health like well child, pregnancy monitoring, cervical cancer screening. Access to this service is appreciated by patients. Despite this, data from the NHIH are showing that there is a reduces number of reported services like pregnancy monitoring and well child and cervical cancer screening. Underreporting is one of the causes but also lack of education of the population in accessing these services.

Point of care testing like lab tests, electrocardiogram (EKG) is not recognised as an offered service in family doctor's office.

Table 5. Health needs in relation with procedures in FD's office 
In the place you live, do you have access to iv injection or infusions? $92,4 \quad 7,6$

In the place you live do you have access to flu- vaccine? $\quad 94,4 \quad 5,6$

In the place you live do you have access to pregnancy monitoring? $\quad 85,6 \quad 14,4$

$\begin{array}{ll}\text { Is your FD doing well child monitoring? } & 90,7 \quad 9,3\end{array}$

In the place you live do you have access to Pap-smear in FD office? $\quad 46,2 \quad 53,8$

In the place you live do you have access to blood draw in FD office? $\quad 63,1 \quad 36,9$

In the place you live do you have access to an electrocardiogram. $\quad 56 \quad 44$

\section{Conclusion}

The analysis of unmet needs in the primary care system in County Brasov, isn't showing a huge gap between patients' expectation and what is offered. It is surprisingly showing a certain degree of contentment of patients with the services offered at this level of care. In our opinion, this mostly reflects the belief of population that the competences of this speciality are limited by its nature and this is how it is supposed to be.

The fact that a medical resource is relatively easily available, encompasses the barriers to access due to limitations in the spectrum of services that is offered. These limitations are merely disturbing for the FDs who are feeling unable to practice their profession. 
FDs seem to be invested by the population as a trusting health resources where information about illnesses and treatment is available. More should be done to validate this important function of the FD.

This study has also revealed some gaps in the provision of primary care from the perspective of normative needs. The primary care system must develop some important attributes like preventive services or continuity of care. Cervical cancer screening is not done in many FDs offices and this is an important public health problem since cervical cancer is highly prevalent in our country.

Health needs assessment would be irrelevant if no implementation plan would follow. The results of our study should be further discussed in the interest groups, between interested parts, inside the profession and between specialties to identify what should be changed in the future contract in order to encourage FDs to practice a broad variety of services and improve the Health of the community.

Limitation of the study

This analysis was done in order to describe the healthcare needs of the population in our region. Generalisability is therefore limited in terms of specific results but some of the conclusions, related to the regulatory policies are generally available.

\section{Declarations}

\section{- List of Abbreviations}

FD: family doctor

NHIS: National Health Insurance System

NHIH: National Health Insurance House 
HNA: Health Needs Assessment

OOH: Out of Hours Services

\section{- Ethics approval and consent to participate}

The study has been approved by the Ethics Committee of Transylvania University. Consent of the patients to participate to the study was verbal and assumed through the completion of the questionnaire. The questionnaire has a heading section that is explaining the reasons of the study and is anonymous. The questionnaire was approved by the Ethics committee.

\section{- Consent for publication}

Not applicable

\section{- Availability of data and materials}

The datasets used and/or analyzed during the current study are available from the corresponding author on reasonable request.

\section{- Competing interests}

The authors declare that they have no competing interests

\section{- Funding}

The project was funded by a research grant offered by Transylvania University of Brasov (Interdisciplinary grant - Responsibility, strategy, prioritization and integrity in regional development of the Health System in County Brasov- Grant number 78/08.01.2018 https://www.unitbv.ro/cercetare/rezultatele-cercetarii/burse-si-granturiunitbv/rapoarte-de-cercetare.html)

\section{- Authors' contributions}

Andrea Neculau is the main author, responsible for the design of the study, ethical committee approval, questionnaire design and validation, data collection, manuscript 
writing and therefore is the corresponding author.

Liliana Rogozea has contributed to study design and data analysis.

Daniela Popa has contributed to questionnaire design and validation

Ioana Atudorei has contributed to questionnaire design and data analysis

Marius Moga has contributed to study design and data analysis.

Florin Leasu has contributed to study design and data analysis

Anca Lacatus has contributed to data analysis and interpretation.

- Acknowledgements

Not applicable

\section{References}

1. State of Health in the EU- România, the Europeam Observatory of Health System and Policies, 2017

2. Vlădescu, Cristian, et al. "Romania: health system review." Health systems in transition18/4 (2016): 1-170.

3. Kleij, Kim-Sarah, et al. "Patients' preferences for primary health care-a systematic literature review of discrete choice experiments." BMC health services research 17.1 (2017): 476 .

4. Wright, John, Rhys Williams, and John R. Wilkinson. "Development and importance of health needs assessment." Bmj7140 (1998): 1310-1313. 
5. Boerma WGW et al. (2012). Evaluation of the organization and provision of primary care in Romania. A survey-based project. Copenhagen: WHO Regional Office for Europe

6. Kringos, Dionne S., et al. Building primary care in a changing Europe. Case studies. WHO Regional Office for Europe, 2015

7. Eurostat- statistic explained- Healthcare activities statistics - consultations, 2014

8. Vlădescu, Cristian, et al. "Romania: health system review." Health systems in transition18/4 (2016): 1-170.

\section{Figures}

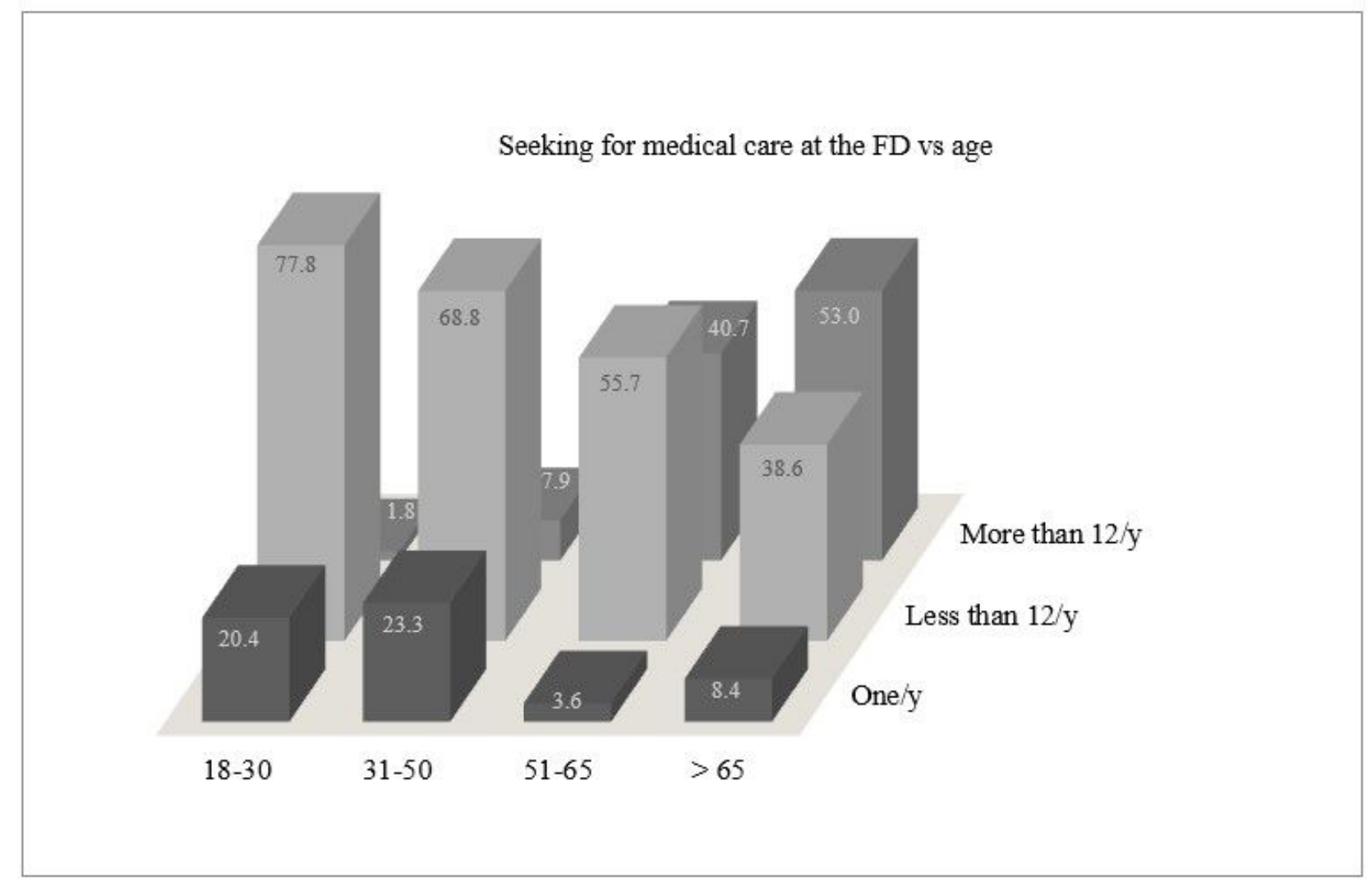




\section{Figure 1}

Seeking for medical care at the FD according to age 